Flat deformation theorem and symmetries in spacetime

This article has been downloaded from IOPscience. Please scroll down to see the full text article.

2009 Class. Quantum Grav. 26055013

(http://iopscience.iop.org/0264-9381/26/5/055013)

The Table of Contents and more related content is available

Download details:

IP Address: 161.116.80.144

The article was downloaded on 17/02/2009 at 15:34

Please note that terms and conditions apply. 


\title{
Flat deformation theorem and symmetries in spacetime
}

\author{
Josep Llosa ${ }^{1}$ and Jaume Carot $^{2}$ \\ ${ }^{1}$ Departament de Física Fonamental, Universitat de Barcelona, Spain \\ ${ }^{2}$ Departament de Física, Universitat de les Illes Balears, Spain
}

Received 11 September 2008, in final form 9 January 2009

Published 17 February 2009

Online at stacks.iop.org/CQG/26/055013

\begin{abstract}
The flat deformation theorem states that given a semi-Riemannian analytic metric $g$ on a manifold, locally there always exists a two-form $F$, a scalar function $c$, and an arbitrarily prescribed scalar constraint depending on the point $x$ of the manifold and on $F$ and $c$, say $\Psi(c, F, x)=0$, such that the deformed metric $\eta=c g-\epsilon F^{2}$ is semi-Riemannian and flat. In this paper we first show that the above result implies that every (Lorentzian analytic) metric $g$ may be written in the extended Kerr-Schild form, namely $\eta_{a b}:=a g_{a b}-2 b k_{(a} l_{b)}$ where $\eta$ is flat and $k_{a}, l_{a}$ are two null covectors such that $k_{a} l^{a}=-1$; next we show how the symmetries of $g$ are connected to those of $\eta$, more precisely; we show that if the original metric $g$ admits a conformal Killing vector (including Killing vectors and homotheties), then the deformation may be carried out in a way such that the flat deformed metric $\eta$ 'inherits' that symmetry.
\end{abstract}

PACS numbers: $04.20 . \mathrm{Cv}, 02.40 . \mathrm{Hw}, 02.40 . \mathrm{Ky}$

\section{Introduction}

It has recently been proved [1] that, given a semi-Riemannian analytic metric $g_{a b}$ on a manifold $\mathcal{M}$, there exist a 2-form $F_{a b}$ and a scalar function $c$ such that

(1) an arbitrary scalar constraint $\Psi\left(c, F_{a b}, x\right)=0, x \in \mathcal{M}$, is fulfilled and

(2) the so-called 'deformed metric'

$$
\eta_{a b}=c g_{a b}-\epsilon F_{a b}^{2} \quad \text { where } \quad \epsilon= \pm 1 \quad \text { and } \quad F_{a b}^{2}:=F_{a c} g^{c d} F_{d b}
$$

is semi-Riemannian and flat

This result was called flat deformation theorem. For the purposes of the present paper, we shall only consider the four-dimensional Lorentzian case.

The proof of the above theorem was based on the existence of solutions for a partial differential system that is derived from the condition that $\eta_{a b}$ is flat. As a consequence of the arbitrariness in the choice of the Cauchy hypersurface and Cauchy data, the deformation (1) 
leading to a flat $\eta_{a b}$ is by no means unique. Furthermore, as the Cauchy-Kovalewski theorem is a cornerstone in the proof, the validity of the theorem is limited to the analytic category.

The purpose of the present paper is to deal with the question of how the symmetries of the metric $g_{a b}$ are reflected upon the deformed metric $\eta_{a b}$, more precisely: assuming that $g_{a b}$ admits a Killing vector field $X^{a}$, we ask whether it is possible to choose $F_{a b}$ and $c$ in (1) such that $X^{a}$ is also a Killing vector field for $\eta_{a b}$. We shall prove that the answer is in the affirmative in the case of non-null Killing vectors and that the symmetry is thus somehow 'inherited' along the deformation.

The paper is structured as follows: section 2 contains some algebraic developments on the consequences of the deformation law (1) for a four-dimensional spacetime which will allow us to state it in a number of alternative ways, thus illustrating different features of the deformation law. In section 3 we present the formalism and prove some intermediate results ${ }^{3}$ in order to demonstrate the theorem alluded to in the previous paragraph. It is worth noticing that in order to prove it, the problem is reformulated on the three-dimensional quotient manifold (see section 4.2), so that a dimensional reduction occurs. Section 5 contains a generalization of the above result to the case of (non-null) conformal Killing vectors. Finally, in section 6, we present some examples which we believe may be of interest due to their physical relevance. We put some technical developments in the appendices in order to make the paper more readable. Also for this reason, we do not insist at every intermediate step on the local character of the results presented here, but the reader should bear this in mind.

\section{Algebraic consequences of the deformation law}

Consider now the 2-form $F_{a b}$ whose existence is granted by the deformation theorem [1]; there are two possibilities, either it is

(a) singular (or null), then, a tetrad $\left\{x_{a}, y_{a}, k_{a}, l_{a}\right\}$ exists such that $g_{a b}=x_{a} x_{b}+y_{a} y_{b}-2 k_{(a} l_{b)}$ and

$$
F_{a b}=2 k_{[a} x_{b]} \quad \text { and then } \quad F_{a b}^{2}=-k_{a} k_{b}
$$

or else it is

(b) non-singular (or non-null), in which case a tetrad such as the one above exists in terms of which $F_{a b}$ reads

$$
F_{a b}=-2 B x_{[a} y_{b]}+2 E k_{[a} l_{b]} \quad \text { and then } \quad F_{a b}^{2}=-B^{2}\left(x_{a} x_{b}+y_{a} y_{b}\right)-2 E^{2} k_{(a} l_{b)}
$$

where $E$ and $B$ are functions related to the algebraic invariants of $F^{a}{ }_{b}:=g^{a c} F_{c b}$. If either $B$ or $E$ is zero, the resulting 2-form is timelike or spacelike respectively. If neither of them vanishes, the 2 -form is said to be non-simple.

In the singular case, the deformation law (1) reads $\eta_{a b}=c g_{a b}+\epsilon k_{a} k_{b}$ or, equivalently,

$$
g_{a b}=\frac{1}{c} \eta_{a b}-\frac{\epsilon}{c} k_{a} k_{b}
$$

with $k_{a} k^{a}=0$ and $\eta_{a b}$ flat. That is, $g_{a b}$ is a conformal Kerr-Schild metric [2]. The singular case is therefore non-generic and encompasses a rather restricted class of metrics.

In the non-singular case, from equations (1) and (3) we have that

$$
\eta_{a b}=a g_{a b}+b S_{a b}
$$

3 This formalism was developed in a number of references, notably [4] and [5] which will be used in section 4. We present it here in a way well suited to our purposes. 
with $a=c+\epsilon B^{2}, b=-\epsilon\left(B^{2}+E^{2}\right)$ and $S_{a b}=-2 k_{(a} l_{b)}$. As was shown in [1], this is the generic case in the sense that the flat deformation (5) can always be achieved for any analytic semi-Riemannian metric.

Note that the arbitrary scalar constraint $\Psi\left(c, F_{a b}, x\right)=0$ has no consequences on the factors $a$ and $b$ in (5). Indeed, including (3) the scalar constraint may be written as $f(c, E, B)=0$ or, equivalently, as a relation $\tilde{f}(c, a, b)=0$ which, at most, can be used to determine $c=c(a, b)$ to choose one amongst the many 2-forms $F_{a b}$ compatible with (5).

We have hitherto proved that:

Proposition 1. Let $g_{a b}$ be a Lorentzian analytic metric on a spacetime $\mathcal{M}$. Locally there exist two scalars, $a$ and $b$, and two null vectors, $k_{a}$ and $l_{a}$, such that $k_{a} l^{a}=-1$ and the metric

$$
\eta_{a b}:=a g_{a b}-2 b k_{(a} l_{b)}
$$

is Lorentzian and flat.

The above expression vaguely recalls a conformal Kerr-Schild transformation, but in the present case two non-parallel null vectors, $k_{a}$ and $l_{a}$, occur. We shall henceforth call this expression extended Kerr-Schild form and proposition 1 can be restated as:

Any Lorentzian analytic metric can be written in the extended Kerr-Schild form.

An equivalent statement is

Proposition 2. Let $g_{a b}$ be a Lorentzian analytic metric on a spacetime $\mathcal{M}$. Locally there exist two scalars, $a$ and $b$, and a hyperbolic two-plane $S_{a b}$ such that the metric

$$
\eta_{a b}:=a g_{a b}+b S_{a b}
$$

is Lorentzian and flat.

Note that $S^{a}{ }_{b}$ is a two-dimensional projector:

$$
S^{a}{ }_{d} S^{d}{ }_{b}=S^{a}{ }_{b}, \quad S^{a}{ }_{a}=2
$$

which projects vectors onto the hyperbolic plane spanned by $\left\{k^{a}, l^{a}\right\}$. If we now denote $H_{a b}:=g_{a b}-S_{a b}$, i.e. the complementary projector, then

$H_{d}^{a} H_{b}^{d}=H_{b}^{a}, \quad H^{a}{ }_{a}=2, \quad$ and $\quad S_{d}^{a} H_{b}^{d}=H_{d}^{a} S_{b}^{d}=0$,

$H_{a b}$ is then the elliptic two-plane spanned by any two spacelike vectors orthogonal to $S_{a b}$, in particular $x^{a}, y^{a}$, the spacelike vectors in the chosen tetrad, i.e. $H_{a b}=2 x_{(a} y_{b)}$, and it is then possible to write the deformation (1) in a way similar to that given by (7) but in terms of the (elliptic) projector $H_{a b}$ instead of the $S_{a b}$, namely

$$
\eta_{a b}:=\bar{a} g_{a b}+\bar{b} H_{a b}
$$

where $\bar{a}$ and $\bar{b}$ are scalars.

From the comments and developments above and taking (7) into account, we can write

$$
g_{a b}:=H_{a b}+S_{a b} \quad \text { and } \quad \eta_{a b}:=(a+b) S_{a b}+a H_{a b},
$$

that is, the almost-product structure [3] defined by $S_{a b}$ is compatible with both metrics, $g_{a b}$ and $\eta_{a b}$, and therefore we can state:

Proposition 3. Let $g_{a b}$ be a Lorentzian analytic metric on a spacetime $\mathcal{M}$. Locally there exists a Lorentzian flat metric $\eta_{a b}$ that shares with $g_{a b}$ an almost-product structure. 


\section{Spacetimes admitting a (non-null) Killing vector}

In this section we are going to set up and develop the formalism and basic results which will later be used in order to prove the result stated in the introduction; namely: that if the metric admits an isometry, it is always possible to preserve it in the flat deformed metric.

Let $\mathcal{M}$ be a spacetime with an arbitrary metric $\eta_{a b}{ }^{4}$ admitting a Killing vector $X^{a}$. Let $\xi_{a}:=\eta_{a b} X^{b}$ and $l:=\xi_{a} X^{a}$. Assume that the Killing is non-null, that is: $l \neq 0$, and denote by $\mathcal{S}$ the set of all orbits of $X^{a}$, which we assume to be a three-manifold (the quotient manifold) ${ }^{5}$.

We shall designate by $\pi$ the canonical projection $\pi: \mathcal{M} \longrightarrow \mathcal{S}$ where $\pi(x)=O_{x}$ is the orbit through the point $x \in \mathcal{M}$ of the one-parameter group generated by $X^{a}$.

The projector,

$$
h_{b}^{a}:=\delta_{b}^{a}-\frac{1}{l} X^{a} \xi_{b}
$$

projects vectors in $T \mathcal{M}$ onto vectors that are transverse (orthogonal) to $X^{a}$. There is a bijection [4] between tensor fields $T_{b \ldots}^{\prime a \ldots}$ on $\mathcal{S}$ and the tensor fields $T_{b \ldots}^{a \ldots}$ on $\mathcal{M}$ that fulfill:

$$
X^{b} T_{b \ldots}^{a \ldots}=0, \quad \xi_{a} T_{b \ldots}^{a \ldots}=0 \quad \text { and } \quad \mathcal{L}_{X} T_{b \ldots}^{a \ldots}=0
$$

that is, those which are transverse to $X^{a}$ and $\xi_{a}$ and Lie invariant along $X^{a}$. Following Geroch [4]: "while it is useful conceptually to have the three-dimensional manifold $\mathcal{S}$, it plays no further logical role in the formalism. We shall hereafter drop the primes: we shall continue to speak of tensor fields being on $\mathcal{S}$, merely as a shorthand way of saying that the field (formally, on $\mathcal{M}$ ) satisfies (13)'.

As $l \neq 0$ the projected metric

$$
h_{a b}:=\eta_{a b}-\frac{1}{l} \xi_{a} \xi_{b}
$$

induces a semi-Riemannian metric on the quotient manifold $\mathcal{S}$, the so-called 'quotient metric'. Its signature is $+1+1-\operatorname{sign}(l)$. We shall designate by $h^{a b}:=\eta^{a b}-\frac{1}{l} X^{a} X^{b}$ the inverse quotient metric, that is: $h^{a b} h_{b c}=h_{c}^{a}$.

\subsection{The Killing equation}

From $\mathcal{L}_{X} \eta_{a b}=0$ it follows that $\nabla_{a} \xi_{b}$ is skew-symmetric, that is: $\nabla_{b} \xi_{a}+\nabla_{a} \xi_{b}=0$ where $\nabla$ stands for the covariant derivative associated with $\eta$.

We also have that $\mathcal{L}_{X} \xi_{a}=0$ and $X^{a} l_{a}=0$, where $l_{a}:=\nabla_{a} l$. Since $X^{a}$ is non-null, $\nabla_{a} \xi_{b}$ can be decomposed as

$2 \nabla_{a} \xi_{b}:=2 f_{[a} \xi_{b]}+\Theta_{a b} \quad$ with $\quad f:=\log |l| \quad$ and $\quad \Theta_{a b} X^{b}=0$.

$\Theta_{a b}=-\Theta_{b a}$ is related to the vorticity of the Killing flow. We shall use the above form for the Killing equation in the following.

\subsection{The Levi-Civita connection on $\mathcal{S}$}

Let $T_{b \ldots}^{a \ldots}$ be a tensor field on $\mathcal{S}$ and define

$$
D_{c} T_{b \ldots}^{a \ldots}:=h_{m}^{a} h_{b}^{n} h_{c}^{k} \nabla_{k} T_{n \ldots}^{m \ldots} .
$$

Clearly, it is a tensor field on $\mathcal{S}$, since $T_{b \ldots}^{a \ldots}$ and $h_{b}^{a}$ both satisfy (13), and, since $X^{a}$ is a KV, the Lie derivative with respect to it commutes with $\nabla$; further it can be easily proved that $D_{a}$ is a

4 Note: $\eta_{a b}$ does not designate the flat metric at this point. We use this notation here for later convenience.

5 It can be shown that locally this is always the case if fixed points of $X^{a}$ are excluded. 
linear connection: indeed, it is linear, it satisfies the Leibniz rule and for any scalar function $f$ on $\mathcal{S}, D_{a} f$ is the gradient of $f$. Moreover, it can also be shown that it is torsion-free and that $D_{c} h_{a b}=0$ (this last result holds trivially); therefore, $D$ is the Levi-Civita connection on $\mathcal{S}$ (see [4]).

Let now $v^{a}, w^{b}$ be two vector fields on $\mathcal{S}$, then taking into account (13), (15) and (16) one easily gets

$$
D_{v} w^{a}=\nabla_{v} w^{a}+\frac{1}{2 l} X^{a} \Theta_{b c} v^{b} w^{c}
$$

where $D_{v} w^{a}:=v^{b} D_{b} w^{a}$. Note the formal similarity between this formula and Gauss equation for hypersurfaces, even though $\mathcal{S}$ is not a submanifold and we have the skew-symmetric $\Theta_{b c}$ instead of the second fundamental form.

\subsection{The Riemann tensor on $\mathcal{S}$}

Consider next a vector field $v^{a}$ on $\mathcal{S}$ endowed with the quotient metric $h_{a b}$ and its associated Levi-Civita connection $D_{a}$ as defined above in (16). We aim at calculating the Riemann tensor $\mathcal{R}_{d a b}^{c}$ for this connection.

From the Ricci identities, $\left[D_{a}, D_{b}\right] v^{c}=v^{d} \mathcal{R}_{d a b}^{c}$, we have that

$$
\mathcal{R}_{a b c d}=R_{a b c d}^{\perp}+\frac{1}{2 l}\left(\Theta_{a b} \Theta_{c d}+\Theta_{[a c} \Theta_{b] d}\right),
$$

where $R_{a b c d}^{\perp}:=h_{a}^{m} h_{b}^{n} h_{c}^{p} h_{d}^{q} R_{m n p q}$. Using the identity $\Theta_{a b} \Theta_{c d}+\Theta_{a c} \Theta_{d b}+\Theta_{a d} \Theta_{b c}=0$ that follows from the fact that $\operatorname{dim} \mathcal{S}=3$, we then arrive at

$$
\mathcal{R}_{a b c d}=R_{a b c d}^{\perp}+\frac{3}{4 l} \Theta_{a b} \Theta_{c d} .
$$

The remaining components of $R_{a b c d}$ follow from the second-order Killing equation [6], $\nabla_{a} \nabla_{b} \xi_{c}=R_{d a b c} X^{d}:=R_{X a b c}$ which, taking into account (15), leads to

$$
\begin{aligned}
& R_{X a b c}^{\perp}=\frac{1}{2} D_{a} \Theta_{b c}+\frac{1}{2} f_{[b} \Theta_{a c]}, \\
& R_{X a X c}=-\frac{1}{2} D_{a} l_{c}-\frac{1}{4} \Theta_{a}{ }^{b} \Theta_{b c}+\frac{1}{4 l} l_{a} l_{c} .
\end{aligned}
$$

We have thus shown that the entire Riemann tensor on $\mathcal{M}$ may be expressed in terms of the kinematic invariants of $\xi_{a}$ and the Riemann tensor on $\mathcal{S}$ associated with the Levi-Civita connection $D_{a}$ of the projected (quotient) metric $h_{a b}$.

\subsection{Lift of a metric from $\mathcal{S}$ to $\mathcal{M}$}

We have hitherto shown how a semi-Riemannian metric can be projected from $\mathcal{M}$ to $\mathcal{S}$. We shall now consider the converse case. As before, let $X^{a}$ be a vector field on $\mathcal{M}$ and let $\mathcal{S}$ be the set of its orbits, which we take to be a manifold according to the reasoning at the beginning of the present section. Further, let $\pi: \mathcal{M} \rightarrow \mathcal{S}$ be the canonical projection.

Let now $h_{a b}$ be a semi-Riemannian metric on $\mathcal{S}$ having constant signature $(++\sigma)$, $\sigma= \pm 1$. We shall denote by the same symbol the pulled back metric on $\mathcal{M}$, i.e.: $\pi^{*} h_{a b}=h_{a b}$, which is degenerate because $h_{a b} X^{b}=0$, moreover, $\mathcal{L}_{X} h_{a b}=0$. The point now is: does a metric $\eta_{a b}$ on $\mathcal{M}$ exist such that: (a) admits $X^{a}$ as a Killing vector and (b) has $h_{a b}$ as the quotient metric? 
If it exists, a relation similar to (14) must hold, with $\xi_{a}:=\eta_{a b} X^{b}$ and $l=\xi_{a} X^{a}$. Hence, the solution is not unique, because we may choose any covector $\xi_{a}$ such that $\mathcal{L}_{X} \xi_{a}=0$ and that $l:=\xi_{a} X^{a}$ has constant sign ${ }^{6}$; then taking

$$
\eta_{a b}:=h_{a b}+\frac{1}{l} \xi_{a} \xi_{b}
$$

as the lifted metric, all the required conditions are satisfied (namely: $X^{a}$ is a KV of $\eta_{a b}$ and $h_{a b}$ is its quotient metric). Then, if no further condition is demanded, equations (18), (19) and (20) merely relate the Riemann tensors for both metrics, $\eta_{a b}$ and $h_{a b}$. However, if we require the lifted metric $\eta_{a b}$ to fulfill some supplementary condition, e.g. to be flat, then these become equations on the chosen $\xi_{a}$ and the given $h_{a b}$, much in the same way as the Gauss curvature equation and the Codazzi-Mainardi equations set up conditions on the way that a submanifold can be immersed in an ambient space.

The choice of $\xi_{a}$ is restricted by the condition $\mathcal{L}_{X} \xi_{a}=0$. Assume that a 1-form $\alpha_{a}$ on $\mathcal{M}$ such that $\alpha_{a} X^{a}=1$ and $\mathcal{L}_{X} \alpha_{a}=0$ is given. Then, the sought $\xi_{a}$ can be written as $\xi_{a}=l\left(\alpha_{a}+\mu_{a}\right)$, with $l:=\xi_{a} X^{a}$ and $\mu_{a} X^{a}=0$. It can easily be proved that

$$
\mathcal{L}_{X} \xi_{a}=0 \quad \Leftrightarrow \quad X l=0 \text { and } \quad \mathcal{L}_{X} \mu_{a}=0 .
$$

Hence, given a 1 -form $\alpha_{a}$ on $\mathcal{M}$ such that $\alpha_{a} X^{a}=1$ and $\mathcal{L}_{X} \alpha_{a}=0$, choosing $\xi_{a}$ is equivalent to choosing a function $l \neq 0$ on $\mathcal{S}$, a 1 -form $\mu_{a}$ on $\mathcal{S}$ and taking $\xi_{a}=l\left(\alpha_{a}+\mu_{a}\right)$.

The exterior derivative of this expression yields

$$
(\mathrm{d} \xi)_{a b}=\frac{2}{l} l_{[a} \xi_{b]}+l(\mathrm{~d} \mu)_{a b} \quad \text { and } \quad \Theta_{a b}=l(\mathrm{~d} \mu)_{a b}+l(\mathrm{~d} \alpha)_{a b}
$$

where (15) has been taken into account.

In terms of $l$ and $\mu_{a}$, taking (22) into account, equations (18), (19) and (20) read, in the special case in which $\alpha_{a}$ is closed:

$$
\begin{aligned}
R_{a b c d}^{\perp} & =\mathcal{R}_{a b c d}-\frac{3 l}{4}(\mathrm{~d} \mu)_{a b}(\mathrm{~d} \mu)_{c d}, \\
R_{X a b c}^{\perp} & =\frac{1}{2} D_{a}\left[l(\mathrm{~d} \mu)_{b c}\right]+\frac{1}{2} l_{[b}(\mathrm{d} \mu)_{a c]}, \\
R_{X a X c} & =-\frac{1}{2} D_{a} l_{c}-\frac{l^{2}}{4}(\mathrm{~d} \mu)_{a d}(\mathrm{~d} \mu)_{b c} h^{b d},
\end{aligned}
$$

which are equations for $l, \mu_{a}$ and $h_{a b}$ to be solved on $\mathcal{S}$.

\subsection{Hypersurfaces and Killing vectors}

Let $\Sigma$ be a surface in $\mathcal{S}$, then $\pi^{-1} \Sigma$ is a hypersurface in $\mathcal{M}$ and the Killing vector $X^{a}$ is tangent to it. The following diagram is commutative:

$$
\begin{aligned}
& (\eta, \nabla, R) \\
& \left(\eta^{\prime}, \nabla^{\prime}, R^{\prime}\right) \\
& (h, D, \mathcal{R}) \\
& \left(h^{\prime}, D^{\prime}, \mathcal{R}^{\prime}\right)
\end{aligned}
$$

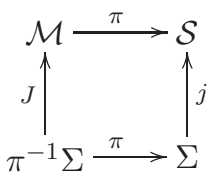

where $J$ and $j$ are the respective embeddings.

6 The sign is to be chosen so that the lifted metric has the required signature $(+++-)$. 
We respectively denote by $\eta_{a b}^{\prime}, \Phi_{a b}, \nabla^{\prime}$ and $R_{a b c d}^{\prime}$ the first and second fundamental forms, the induced connection and the intrinsic curvature on $\pi^{-1} \Sigma$ as a hypersurface of the Riemannian manifold $\left(\mathcal{M}, \eta_{a b}\right)$. Similarly, we denote by $h_{a b}^{\prime}, \phi_{a b}, D^{\prime}$ and $\mathcal{R}_{a b c d}^{\prime}$ the corresponding objects on $\Sigma$ regarded as a hypersurface in $\left(\mathcal{S}, h_{a b}\right)$.

Let $n^{a}$ be the unit vector $\eta$-normal to $\pi^{-1} \Sigma$. Since $X^{a}$ is tangent to $\pi^{-1} \Sigma$, then $\xi_{a} n^{a}=0$. Furthermore, $\mathcal{L}_{X} n^{a}=0$. Indeed, for any $V^{a}$ tangent to $\pi^{-1} \Sigma$ we have that $\mathcal{L}_{X} V^{a}$ is also tangent to $\pi^{-1} \Sigma$ and, using that $X^{a}$ is a Killing vector field, we easily arrive at $\eta_{a b} \mathcal{L}_{X} n^{a} V^{b}=0$, which implies that $\mathcal{L}_{X} n^{a} \propto n^{a}$. On the other hand, as $n^{a}$ is unit, $\eta_{a b} \mathcal{L}_{X} n^{a} n^{b}=0$, whence it follows that $\mathcal{L}_{X} n^{a}=0$. Therefore, $n^{a}$ is also a vector in $\mathcal{S}$ and is the unit vector $h$-normal to $\Sigma$.

It can easily be proved that the second fundamental forms for $\pi^{-1} \Sigma$ and $\Sigma$ satisfy that: $\phi_{a b}=\Phi_{a b}^{\perp}$. On the other hand, for any vector field $V^{b}$ tangent to $\pi^{-1} \Sigma$, we have that

$$
\Phi_{a b} X^{a} V^{b}=\nabla_{X} n_{b} V^{b}=-\nabla_{V} \xi_{b} n^{b}=-\frac{1}{2}(\mathrm{~d} \xi)_{a b} V^{a} n^{b}
$$

where in the second equality we have used that $\mathcal{L}_{X} V^{a} n_{a}=0$ and that $V^{b} n_{b}=0$. The above equation implies, putting $(\mathrm{d} \xi)_{a b} n^{b}:=(\mathrm{d} \xi)_{a n}$ and $f_{b} n^{b}:=f_{n}$,

$$
\Phi_{a b} X^{a}=\frac{1}{2}(\mathrm{~d} \xi)_{n b}=\frac{1}{2} f_{n} \xi_{b}+\frac{1}{2} \Theta_{n b}
$$

where (15) has been taken into account. Therefore,

$$
\Phi_{a b}=\phi_{a b}+\frac{1}{2 l} f_{n} \xi_{a} \xi_{b}+\frac{1}{l} \Theta_{n(a} \xi_{b)}
$$

\section{Flat deformation}

The aim of this section is to prove the main result in this paper, namely,

Theorem 1. Let $\left(\mathcal{M}, g_{a b}\right)$ be a spacetime with a metric $g_{a b}$ admitting a non-null Killing vector $X^{a}$. Locally there exists a deformation law

$$
\eta_{a b}=a g_{a b}+b H_{a b}
$$

where $a$ and $b$ are two scalars, $H_{a b}$ is a two-dimensional projector on a g-elliptic plane and $\eta_{a b}$ is flat and also admits $X^{a}$ as a Killing vector.

It will be convenient for our purposes to prove the following result previously:

Proposition 4. Let $X^{a}$ be a Killing vector for $g_{a b}$ and let $\eta_{a b}$ be defined by (28) with $b \neq 0$, then

$$
\mathcal{L}_{X} \eta_{a b}=0 \quad \Leftrightarrow \quad \mathcal{L}_{X} a=\mathcal{L}_{X} b=0 \quad \text { and } \quad \mathcal{L}_{X} H_{a b}=0 .
$$

Proof. As $\mathcal{L}_{X} g_{a b}=0, \mathcal{L}_{X} \eta_{a b}=0$ implies that

$$
\mathcal{L}_{X} a g_{a b}+\mathcal{L}_{X} b H_{a b}+b \mathcal{L}_{X} H_{a b}=0 .
$$

Since $H_{b}^{a}$ is a two-dimensional projector, $H^{a b} H_{a b}=H_{a}^{a}=2$, and taking the Lie derivative we get $2 \mathcal{L}_{X} H_{a b} H^{a b}=0$. Contraction of (30) with $g^{a b}$ and $H^{a b}$ leads respectively to

$$
4 \mathcal{L}_{X} a+2 \mathcal{L}_{X} b=0 \quad \text { and } \quad 2 \mathcal{L}_{X} a+2 \mathcal{L}_{X} b=0
$$

which imply: $\mathcal{L}_{X} a=\mathcal{L}_{X} b=0$. Substituting back into (30) and taking into account that $b \neq 0$ yields $\mathcal{L}_{X} H_{a b}=0$.

The proof of theorem 1 spreads over the present section and it consists of finding $a, b$ and $H_{a b}$ such that 
(i) $\eta_{a b}=a g_{a b}+b H_{a b}$ is flat and

(ii) $\mathcal{L}_{X} a=\mathcal{L}_{X} b=0$ and $\mathcal{L}_{X} H_{a b}=0$.

The number of unknowns is 6, namely: 2 for $a$ and $b$ plus 4 for $H_{a b}$ (recall the constraints $H_{c}^{a} H_{b}^{c}=H_{b}^{a}$ and $H_{a}^{a}=2$ ). Then, (i) means that the Riemann tensor for $\eta_{a b}$ vanishes,

$$
R_{a b c d}=0 \text {. }
$$

To ensure (ii) we shall solve (31) on $\mathcal{S}$ and then pull the solutions back to $\pi^{-1} \mathcal{S}=\mathcal{M}$.

We first introduce the decompositions:

$$
g_{a b}=p_{a b}+\frac{1}{\bar{l}} \bar{\xi}_{a} \bar{\xi}_{b} \quad \text { and } \quad \eta_{a b}=h_{a b}+\frac{1}{l} \xi_{a} \xi_{b}
$$

where $\bar{\xi}_{a}:=g_{a b} X^{b}$ and $\bar{l}=\bar{\xi}_{b} X^{b}$ are known from the data $g_{a b}$ and $X^{b}$, whereas

$$
\xi_{a}:=\eta_{a b} X^{b}=a \bar{\xi}_{a}+b H_{a b} X^{b} \quad \text { and } \quad l:=\xi_{a} X^{a},
$$

depend on the unknowns. Note that $b H_{a b} X^{a} X^{b}=l-a \bar{l}$.

\subsection{The projection of our problem onto the quotient manifold $\mathcal{S}$}

We must now replace the unknowns $\left(a, b, H_{a b}\right)$, which are tensor quantities on $\mathcal{M}$, with others that are tensor quantities on $\mathcal{S}$. Consider the covector $\alpha_{a}=\bar{\xi}_{a} / \bar{l}$. It is obvious that $\alpha_{a} X^{a}=1$ and $\mathcal{L}_{X} \alpha_{a}=0$; hence the results in section 3.4 can be applied and we have that $\mu_{a}=\frac{1}{l} \xi_{a}-\frac{1}{\bar{l}} \bar{\xi}_{a}$ is a covector in $\mathcal{S}$, thus we can write

$$
\xi_{a}=\operatorname{lm} v_{a}+\frac{l}{\bar{l}} \bar{\xi}_{a}
$$

where $v_{a}$ is a $p$-unitary covector on $\mathcal{S}$ and $m:=\sqrt{\mu_{a} \mu_{b} p^{a b}}$. Then, on account of (33), we have that ${ }^{7}$

$$
H_{a b} X^{b}=\frac{1}{b}\left(l m v_{a}+\frac{l-a \bar{l}_{\bar{l}}}{\bar{l}} \bar{\xi}_{a}\right) .
$$

Now, $H^{a}{ }_{b}$ is a two-dimensional projector and therefore its eigenvalues are 0 and 1 , both with multiplicity 2. $H^{a}{ }_{b} X^{b}$ is an eigenvector (not unit), and a second one may be chosen so that it is $g$-orthogonal to it. We can thus write

$$
H_{a b}=\beta_{a} \beta_{b}+\omega_{a} \omega_{b},
$$

where $\beta_{a}$ and $\omega_{a}$ are $g$-unitary and mutually $g$-orthogonal, and

$$
\beta_{a}=\frac{l m}{\sqrt{b(l-a \bar{l})}} v_{a}+\frac{1}{\bar{l}} \sqrt{\frac{l-a \bar{l}}{b}} \bar{\xi}_{a} .
$$

Since $H^{a}{ }_{b}$ is a projector, it follows that $\omega_{a} X^{a}=\omega_{a} \nu^{a}=0$, and as $\beta_{a}$ is $g$-unitary we also have that

$$
\frac{l^{2} m^{2}}{l-a \bar{l}}=b+a-\frac{l}{\bar{l}} .
$$

From $\mathcal{L}_{X} H_{a b}=0$ (proposition 4), its transverse projection

$$
\tilde{H}_{a b}=\frac{(b+a) \bar{l}-l}{b \bar{l}} v_{a} v_{b}+\omega_{a} \omega_{b}
$$

satisfies also $\mathcal{L}_{X} \tilde{H}_{a b}=0$. Hence, $\tilde{H}_{a b}$ is a tensor on $\mathcal{S}$.

7 We explicitly exclude the cases $l-a \bar{l}=0$ and $b=0$ since they are non-generic. Note that $b=0$ corresponds to the metric $g$ being conformally flat. 
The quotient metric $h_{a b}$ is the transverse projection of $\eta_{a b}$ and, taking (28), (32) and (39) into account, we obtain

$$
h_{a b}=a p_{a b}+\left(b+a-\frac{l}{\bar{l}}\right) v_{a} v_{b}+b \omega_{a} \omega_{b} .
$$

We have seen so far that the set of unknowns $\left\{a, b, H_{a b}\right\}$-tensor quantities on $\mathcal{M}-$ can be assigned the new set of unknowns $\left\{a, b, l, v_{a}, \omega_{b}\right\}$, where $v_{a}$ and $\omega_{b}$ are $p$-unitary and mutually $p$-orthogonal covectors on $\mathcal{S}$, and $a, b$ and $l$ are scalar functions on $\mathcal{S}$. The inverse correspondence is easily established. It suffices to take $H_{a b}$ as defined by (36) with $\beta$ defined by (37).

(Note that the number of degrees of freedom is still 6 because, once $v_{a}$ is given, the unit orthogonal covector $\omega_{a}$ is determined by only giving one angle.)

Due to the symmetries of the Riemann tensor, $R_{a b c d}$, it can be separated as

$$
R_{a b c d}=L_{a b c d}+\frac{2}{l}\left(L_{a b[c} \xi_{d]}+L_{c d[a} \xi_{b]}\right)+\frac{4}{l^{2}} \xi_{[b} L_{a][c} \xi_{d]}
$$

where $L_{a b c d}, L_{a b c}$ and $L_{a c}$ are transverse to $X^{b}$ and have the following symmetries:

(a) $L_{a b c d}$ has the same symmetries as a Riemann tensor in three dimensions,

(b) $L_{a b c}=-L_{b a c}, L_{a b c}+L_{b c a}+L_{c a b}=0$ and $L_{a b}=L_{b a}$.

Note that:

$$
L_{a b c d}=R_{a b c d}^{\perp}, \quad L_{a b c}=R_{a b c X}^{\perp} \quad \text { and } \quad L_{a c}=R_{X a X c}
$$

and are given by (18), (19) and (20). Then equations (31) - flatness of $\eta_{a b}$-are equivalent to

$$
L_{a b c d}=0, \quad L_{a b c}=0 \quad \text { and } \quad L_{a c}=0 .
$$

By taking the exterior differential of $m v_{a}$ and taking (34) and (22) into account, we have that

$$
2 D_{[a}\left(m v_{b]}\right)=\frac{1}{l} \Theta_{a b}-\frac{1}{\bar{l}} \bar{\Theta}_{a b}
$$

with $m$ given by (38). Including now (18), (19), (20), (40) and (44), equations (43) result in second-order partial differential equations relating $a, b, l, v_{a}$ and $\omega_{b}$, i.e. tensor quantities on $\mathcal{S}$.

\subsection{The constraints and the reduced system}

Equations (43) constitute a system of 20 independent equations for only 6 independent unknowns. To handle this overdetermination we shall take six equations among them as a reduced partial differential system (PDS) [7], which we shall solve by giving Cauchy data on a non-characteristic surface $\Sigma$ [8]. The remaining 14 equations are to be considered as constraints to be fulfilled by the Cauchy data on $\Sigma$. It must then be proved that any given solution of the reduced PDS fulfilling the constraints on $\Sigma$ also fulfils them on a neigbourhood of $\Sigma$.

Given a surface $\Sigma \subset \mathcal{S}$, we choose Gaussian $p$-normal coordinates $\left(x^{1}, x^{2}, x^{3}\right)$ on a neighbourhood $\mathcal{U} \subset \mathcal{S}$ of $\Sigma$ :

$x^{1}=0$ on $\Sigma, \quad p_{11}=s= \pm 1 \quad$ and $\quad p_{1 j}=0, \quad j=2,3$.

The sign $s$ depends on the sign of $\bar{l}$ : if $\bar{l}<0$, then $s=+1$, while for $\bar{l}>0, s$ can take both values \pm 1 . For the sake of simplicity, here we shall choose $\Sigma$ so that $s=-\operatorname{sign}(\bar{l})$ and then $p_{i j}$ has signature $(++)$. 
In these coordinates, we choose (indices $a, b, c, \ldots$ run from 1 to 3 and $i, j, \ldots$ run from 2 to 3 )

$$
L_{11}=0, \quad L_{1 j 1}=0, \quad L_{1 i 1 j}=0
$$

as the reduced partial differential system and

$$
L_{a j}=0, \quad L_{b i j k}=0, \quad L_{j c d}=0
$$

as the constraints. (Note that $L_{1 j k}=0$ is included in the above equalities because, as a consequence of the first Bianchi identity, $L_{1 j k}=-L_{j k 1}-L_{k 1 j}$.)

In appendix $\mathrm{A}$ we prove that, if $a, b, H_{a b}$ is an analytic solution of the reduced PDS (46) fulfilling the constraints (47) on $\Sigma$, then the constraints are also fulfilled in an open neigbourhood of $\Sigma$.

\subsection{The reduced PDS}

We shall now write equations (46) in terms of the unknowns $\left\{a, b, l, v_{a}, \omega_{a}\right\}$. We shall only make explicit the principal parts, i.e. those terms involving the second-order partial derivatives with respect to the coordinate $x^{1}$. In what follows a 'dot' will stand for $\partial_{1}$, whereas $\cong$ will mean 'equal apart from non-principal terms'.

(a) From (42) and (20), and taking into account that $l \neq 0$, we have that $L_{11}=0$ leads to

$$
\ddot{l} \cong 0 \text {. }
$$

(b) From (42) and (19), including (44), we obtain $L_{a b c} \cong-D_{c}\left[l D_{[a}\left(m v_{b]}\right)\right]$. Therefore, $L_{1 j 1}=0$ amounts to

$$
\ddot{m} v_{j}+m \ddot{v}_{j} \cong 0, \quad j=2,3
$$

with $m$ given by (38).

(c) From (42) and (18) we have that the third of the equations (46) $L_{1 i 1 j}=0$ leads to

$$
\ddot{h}_{i j} \cong 0
$$

which, using (39), (40), (48) and (49), becomes

$$
\ddot{a}\left(p_{i j}+[\bar{l}(b+a)-l] v_{i} v_{j}\right)+\ddot{b} \omega_{i} \omega_{j}+b\left[\ddot{\omega}_{i} \omega_{j}+\omega_{i} \ddot{\omega}_{j}\right] \cong 0, \quad i, j=2,3 .
$$

The characteristic determinant for the reduced partial differential system constituted by the six equations (48), (49) and (51) is (see appendix B for details)

$$
\begin{aligned}
\Delta:=2 b \omega_{1}^{2} v_{1} \tau_{1} p & {\left[1-s \omega_{1}^{2}+[\bar{l}(b+a)-l] v_{1}^{2}\right] \frac{l-a \bar{l}}{l^{2}} } \\
\times & {\left[\left(b+a-\frac{l}{\bar{l}}\right)\left(1-s v_{1}^{2}\right)-s \omega_{1}^{2}\left(a-\frac{l}{\bar{l}}\right)\right] . }
\end{aligned}
$$

\subsection{Geometrical meaning of the constraints}

It remains to be shown that Cauchy data fulfilling the constraints (47) on the Cauchy surface $\Sigma$ do exist. Consider $\pi^{-1} \Sigma$, which is a hypersurface in $\mathcal{M}$, and take coordinates $\left(x^{1}, \ldots, x^{4}\right)$ adapted to both $X^{a}$ and $\pi^{-1} \Sigma$, i.e. $X^{a}=\delta_{4}^{a}$ and $x^{1}=0$ on $\pi^{-1} \Sigma$.

Let $\bar{P}_{b}^{a}$ and $P_{b}^{a}$ be the projectors

$$
\bar{P}_{b}^{a}:=\delta_{b}^{a}-\frac{1}{g^{11}} g^{1 a} \delta_{b}^{1} \quad \text { and } \quad P_{b}^{a}:=\delta_{b}^{a}-\frac{1}{\eta^{11}} \eta^{1 a} \delta_{b}^{1} .
$$

10 
They both project vectors in $T \mathcal{M}$ onto the hyperplane $T\left(\pi^{-1} \Sigma\right)$ and, while $\bar{P}_{b}^{a}$ projects parallelly to $g^{1 a}, P_{b}^{a}$ does it parallelly to $\eta^{1 a}$. It is obvious that $\bar{P}_{b}^{1}=P_{b}^{1}=0$, hence

$$
\bar{P}_{b}^{a} P_{c}^{b}=P_{c}^{a} \quad \text { and } \quad P_{b}^{a} \bar{P}_{c}^{b}=\bar{P}_{c}^{a}
$$

which implies that, when restricted to the hyperplane $T\left(\pi^{-1} \Sigma\right)$, both projectors, $\bar{P}_{b}^{a}$ and $P_{b}^{a}$, yield the identity.

It is easy to see that the constraints (47) amount to

$$
R_{a b c d}=0 \quad \text { whenever at most one of the indices is } 1
$$

that is, $R_{a b c d} \bar{P}_{e}^{b} \bar{P}_{f}^{c} \bar{P}_{g}^{d}=0$ which, including (53) is equivalent to

$$
R_{a b c d} P_{e}^{b} P_{f}^{c} P_{g}^{d}=0 .
$$

Then, if $n^{a}$ is the unit vector $\eta$-normal to $\pi^{-1} \Sigma$, (54) is equivalent to

$$
R_{a b c d}^{\mathrm{tang}}=0 \quad \text { and } \quad R_{n b c d}^{\mathrm{tang}}=0,
$$

where 'tang' denotes components tangential to $\pi^{-1} \Sigma$ and $R_{n b c d}:=R_{a b c d} n^{a}$.

$\pi^{-1} \Sigma$ can be seen both as a hypersurface of the Riemannian manifold $\left(\mathcal{M}, \eta_{a b}\right)$ and as a hypersurface of $\left(\mathcal{M}, g_{a b}\right)$. We shall denote $\eta_{a b}^{\prime}$ and $g_{a b}^{\prime}$ the respective first fundamental forms. The two normal vectors are respectively:

$n^{a}=\frac{1}{\sqrt{\left|\eta^{11}\right|}} \eta^{1 a}, \quad n_{a}=\frac{1}{\sqrt{\left|\eta^{11}\right|}} \delta_{a}^{1} \quad$ and $\quad \bar{n}^{a}=\frac{1}{\sqrt{\left|g^{11}\right|}} g^{1 a}, \quad \bar{n}_{a}=\frac{1}{\sqrt{\mid g^{11 \mid}}} \delta_{a}^{1}$

and the second fundamental forms are

$$
\Phi_{a b}=P_{a}^{c} \nabla_{c} n_{b} \quad \text { and } \quad \bar{\Phi}_{a b}=\bar{P}_{a}^{c} \bar{\nabla}_{c} \bar{n}_{b} .
$$

The Gauss curvature equation for $\pi^{-1} \Sigma$ as a submanifold of $\left(\mathcal{M}, \eta_{a b}\right)$ reads [9]:

$$
R_{a b c d}^{\mathrm{tang}}=R_{a b c d}^{\prime}+2 \Phi_{a[d} \Phi_{c] b}
$$

and the Codazzi-Mainardi equation is

$$
R_{n b c d}^{\mathrm{tang}}=2 \nabla_{[d}^{\prime} \Phi_{c] b}
$$

where $\nabla^{\prime}$ and $R_{a b c d}^{\prime}$ are respectively the induced connection and the intrinsic curvature.

The constraints (55) are therefore equivalent to

$$
R_{a b c d}^{\prime}+2 \Phi_{a[d} \Phi_{c] b}=0 \quad \text { and } \quad \nabla_{[d}^{\prime} \Phi_{c] b}=0
$$

a particular solution of which is

$$
\Phi_{a b}=0 \quad \text { and } \quad R_{a b c d}^{\prime}=0 .
$$

The normal derivatives of the unknowns. The first of equations (59) determines the first-order normal derivatives of the unknowns on the Cauchy hypersurface $\Sigma$. Indeed, from (27) and $\Phi_{a b}=0$ we have that

$$
f_{n}=0, \quad \Theta_{n b}=0, \quad \phi_{a b}=0 .
$$

Furthermore, as $\Theta_{a b}$ is skew-symmetric and $\phi_{a b} n^{b}=0$, it is obvious that $\phi_{a b}=0$ and $\Theta_{n a}=0$ are equivalent to

$$
\phi_{i j}=0 \quad \text { and } \quad \Theta_{n j}=0, \quad i, j=2,3 .
$$

Note that the remaining equations, namely $\phi_{a 4}=0$ and $\Theta_{n 4}=0$, are identically satisfied because $\phi_{a b}$ and $\Theta_{a b}$ are tensors on $\mathcal{S}$ and in these coordinates $X^{a}=\delta_{4}^{a}$. 
Including then (27), (33) and (44), equations (60) are equivalent to

$$
n^{b} D_{b} l=0, \quad 2 l \bar{D}_{[b}\left(m v_{j]}\right) n^{b}+\frac{l}{\bar{l}} \bar{\Theta}_{n j}=0, \quad D_{i} n_{j}=0
$$

and, using (56), we have that

$$
D_{a} n_{b}=\sqrt{\frac{\left|g^{11}\right|}{\left|\eta^{11}\right|}}\left(\bar{\phi}_{a b}+\frac{1}{2} \bar{n}_{b} D_{a} \log \left[\frac{\left|g^{11}\right|}{\left|\eta^{11}\right|}\right]-b_{a b}^{c} \bar{n}_{c}\right),
$$

where $b_{a b}^{c}$ is the difference tensor for the connections $D$ and $\bar{D}$.

In Gaussian $p$-normal coordinates, taking into account (40) and writing explicitly the principal terms only, (61) becomes

$$
h^{11} \dot{l} \cong 0, \quad h^{11}\left(\dot{m} v_{j}+m \dot{v}_{j}\right) \cong 0, \quad h^{11} \dot{h}_{i j} \cong 0
$$

The similitude of these equations with (48), (49) and (50) is apparent and the characteristic determinant is $\left(h^{11}\right)^{6} \Delta$. Hence, provided that the Cauchy data on $\Sigma$ are chosen so that $\Delta \neq 0$ and $h^{11} \neq 0$, the constraints $\Phi_{a b}=0$ permit to obtain the first order normal derivatives of the unknowns, namely $\dot{a}, \dot{b}, \dot{l}, \dot{v}_{b}$ and $\dot{\omega}_{c}$ on $\Sigma$, in terms of the values of $a, b, l, v_{b}$ and $\omega_{c}$ on $\Sigma$.

The unknowns on the Cauchy surface $\Sigma$. The second of equations in (59) is a condition on the values of the unknowns on $\Sigma$. The isometry group $G$ generated by $X^{a}$ acts also on $\pi^{-1} \Sigma$ and $\pi^{-1} \Sigma / G=\Sigma$. Hence, relations similar to (18)-(20) hold

$$
\begin{aligned}
& R_{a b c d}^{\prime \perp}=\mathcal{R}_{a b c d}^{\prime}-\frac{1}{2 l}\left(\Theta_{a b}^{\prime} \Theta_{c d}^{\prime}+\Theta_{[a c}^{\prime} \Theta_{b] d}^{\prime}\right)=0 \\
& R_{X a b c}^{\prime \perp}=\frac{1}{2} D_{a}^{\prime} \Theta_{b c}^{\prime}+\frac{1}{2} f_{[b}^{\prime} \Theta_{a c]}^{\prime}=0 \\
& R_{X a X c}^{\prime}=-\frac{1}{2} D_{a}^{\prime} l_{c}^{\prime}-\frac{1}{4} \Theta_{a}^{\prime b} \Theta_{b c}^{\prime}=0
\end{aligned}
$$

with $R^{\prime}:=J^{*} R, \mathcal{R}^{\prime}:=j^{*} \mathcal{R}, \Theta^{\prime}:=j^{*} \Theta, l^{\prime}=j^{*} l$.

As $\Sigma$ has only two dimensions, $\Theta_{a c}^{\prime} \Theta^{\prime b c}=\theta^{\prime 2} h^{\prime b}{ }_{a}$, where $2 \theta^{\prime 2}=\Theta_{b c}^{\prime} \Theta^{\prime b c}$. Hence, equation (64) is equivalent to $\Theta^{\prime b c} R_{X a b c}^{\prime \perp}=0$ which, after a little algebra yields $D_{a}^{\prime} \theta^{\prime 2}+f_{a}^{\prime} \theta^{\prime 2}=$ 0 and, since $f^{\prime}=\log \left|l^{\prime}\right|$, we have that

$$
\theta^{\prime 2} l^{\prime}=\text { constant. }
$$

In two dimensions, the Riemann tensor has only one independent component: $\mathcal{R}_{a b c d}^{\prime}=$ $\mathcal{R}^{\prime} h_{a[c}^{\prime} h_{d] b}^{\prime}$, therefore (63) and (65) are respectively equivalent to

$$
\mathcal{R}^{\prime}=\frac{3 \theta^{\prime 2}}{2 l^{\prime}} \quad \text { and } \quad D_{a}^{\prime} D_{c}^{\prime} l^{\prime}=\frac{1}{2} \theta^{\prime 2} h_{a c}^{\prime} .
$$

The integrability conditions for this equation imply that $\theta^{\prime}=0$. Indeed, as

$$
D_{b}^{\prime} D_{a}^{\prime} D_{c}^{\prime} l^{\prime}-D_{a}^{\prime} D_{b}^{\prime} D_{c}^{\prime} l^{\prime}=-\mathcal{R}_{c b a}^{\prime d} D_{d}^{\prime} l^{\prime}
$$

we have that $D_{[b}^{\prime} \theta^{\prime 2} h_{a] c}^{\prime}=-\mathcal{R}^{\prime} D_{[b}^{\prime} l^{\prime} h_{a] c}^{\prime}$, where the fact that we are in two dimensions has been used to simplify the Riemann tensor. Now taking into account the first equation (67) we obtain $D_{b}^{\prime} \theta^{\prime 2}-\frac{3 \theta^{\prime 2}}{2 l^{\prime}} D_{b}^{\prime} l^{\prime}$, or $\theta^{\prime 2} / l^{\prime 3}=$ constant or $\theta^{\prime 2} l^{\prime-3}=$ constant. This, together with (66) implies $l^{\prime}=$ constant which substituted into (67) leads to $\theta^{\prime}=0$.

Therefore, equations (63)-(65) are equivalent to

$$
\mathcal{R}^{\prime}=0, \quad \theta^{\prime}=0 \quad \text { and } \quad D_{a}^{\prime} D_{c}^{\prime} l^{\prime}=0 .
$$

The Gaussian $p$-normal coordinates introduced in section 4.2, equation (45), are especially well suited to our problem. In these coordinates vectors that are tangent to $\Sigma$ are characterized 
by $v^{1}=0$ and the restriction to $\Sigma$ of any covariant tensor on $\mathcal{S}, T_{a b \ldots}, a, b, \ldots=1,2,3$, merely consists in keeping the components $T_{i j \ldots}, i, j, \ldots=2,3$. Thus, $h_{i j}^{\prime}:=\left(j^{*} h\right)_{i j}=$ $h_{i j}, v_{i}^{\prime}:=\left(j^{*} v\right)_{i}=v_{i}, \bar{\Theta}_{i j}^{\prime}:=\left(j^{*} \bar{\Theta}\right)_{i j}=\bar{\Theta}_{i j}, m^{\prime}:=m \circ j=m$ and so on.

Now, including this and the second equation (68), the restriction to $\Sigma$ of equation (44) is

$$
2 D_{[i}^{\prime}\left(m v_{j]}\right)=-\frac{1}{\bar{l}} \bar{\Theta}_{i j}, \quad i, j=2,3
$$

and, as all differential forms in $\Lambda^{2} \Sigma$ are closed, this equation is locally integrable and yields $m v_{j}, j=2,3$.

Moreover, $l^{\prime}=$ constant is a solution of the third equation (68) and therefore we shall take $l=$ constant on $\Sigma$.

As $\Sigma$ has only two dimensions, $\mathcal{R}^{\prime}=2 \epsilon^{\prime i j}\left(h^{\prime}\right) \epsilon^{\prime k l}\left(h^{\prime}\right) \mathcal{R}_{i j k l}^{\prime}$, where $\epsilon^{\prime i j}\left(h^{\prime}\right)$ is the volume tensor on $\Sigma$ for the metric $h_{k l}^{\prime}$. In two dimensions the volume tensors $\epsilon^{\prime i j}\left(h^{\prime}\right)$ and $\epsilon^{\prime i j}\left(p^{\prime}\right)$ are proportional to each other and therefore $\mathcal{R}^{\prime}=0$ is equivalent to $\epsilon^{\prime i j}\left(p^{\prime}\right) \epsilon^{\prime k l}\left(p^{\prime}\right) \mathcal{R}_{i j k l}^{\prime}=0$, or

$$
p^{i k} p^{j l} \mathcal{R}_{i j k l}^{\prime}=0 \text {. }
$$

This is a condition on $h_{i j}^{\prime}$ which depends on the unknowns $a, b, l, v_{a}, \omega_{b}, a, b=1,2,3$.

From the third equation (68) we know that $l=$ constant on $\Sigma$. Then, by solving equation (69) we obtain $m v_{j}, j=2,3$, on $\Sigma$. We then choose $\omega_{i}, i=2,3$, on $\Sigma$ which, together with the orthogonality conditions

$$
\omega_{a} \omega_{b} p^{a b}=v_{a} v_{b} p^{a b}=1 \quad \text { and } \quad v_{a} \omega_{b} p^{a b}=0,
$$

permit to obtain $\omega_{b}, v_{a}, a, b=1,2,3$ and $m$. Finally, substituting this into (38), we can obtain $b=b(a)$ and therefore condition (70) yields a partial differential equation for $a$, whose principal part is

$$
\left(v^{j} v^{k}-\left[1+p^{i l} v_{i} v_{l}\right] p^{j k}\right) \partial_{j k} a \cong 0 \quad \text { where } \quad v^{j}:=p^{j k} v_{k} .
$$

The characteristic form is

$$
\chi\left(z_{l}\right)=\left(z_{l} v^{l}\right)^{2}-\left[1+p^{i l} v_{i} v_{l}\right] p^{j k} z_{j} z_{k}
$$

and the existence of non-characteristic lines for equation (70) is obvious.

\subsection{Summary of the proof}

So far, we have analyzed the existence of a solution to the problem stated in section 1 . Let us now synthesize a way to find such a solution:

(a) from the given $X^{a}$ and $g_{a b}$, obtain $\bar{l}, \bar{\xi}_{a}, \bar{\Theta}_{a b}$ and the quotient metric $p_{a b}$;

(b) choose a Cauchy surface $\Sigma \stackrel{j}{\rightarrow} \mathcal{S}$ and a chart of Gaussian $p$-normal coordinates for $\Sigma,\left(x^{1}, x^{2}, x^{3}\right)$

(c) choose $m v_{i}, i=2,3$, on $\Sigma$ as a solution of $2 \bar{l} \partial_{[i}\left(m v_{j]}\right)=-\bar{\Theta}_{i j}$;

(d) take $l=$ constant on $\Sigma$;

(e) then choose $\omega_{i}, i=2,3$, such that inequality $\Delta \neq 0$ is fulfilled and, including the orthonormality condition, the definition (33) and the obtained value for $m v_{j}$, derive $\omega_{1}, v_{1}$ and $m$ on $\Sigma$;

(f) with the relation (38) obtain $b=b(a)$ and

(g) substitute the above into (70) and solve it to obtain $a$ on $\Sigma$.

With this we have $a, b, l, v_{c}, \omega_{d}$ on $\Sigma$. Then

(a) solve (61) to derive $\dot{a}, \dot{b}, \dot{l}, \dot{v}_{c}, \dot{\omega}_{d}$ on $\Sigma$ and

(b) with these Cauchy data, solve the reduced partial differential system (46); then use (34) to have $\xi_{a}$, (40) to have $h_{a b}$ and (32) to have $\eta_{a b}$. 


\section{Generalization to conformal Killing vectors}

The main result in this paper, stated in theorem 1, can be extended almost immediately to the case of conformal Killing vectors ( $\mathrm{CKV}$ for short), as a consequence of the so called Defrise-Carter's theorem (see for instance [11]); which states, roughly speaking, that given a (non-conformally flat) metric $g$ admitting an $r$-dimensional Lie algebra of CKVs, $C_{r}$, there exists a function $\Omega$, such that $C_{r}$ becomes a Lie algebra of Killing vectors for the conformally related metric $\tilde{g}=\Omega^{2} g$.

Thus, we can state:

Theorem 2. Let $(\mathcal{M}, g)$ be a spacetime such that the metric $g_{a b}$ admits a non-null $C K V X^{a}$. Locally, there exists a deformation law as the one given by (28) such that $X^{a}$ is a KV for the flat metric $\eta_{a b}$.

Proof. Since $X^{a}$ is a CKV of the metric $g_{a b}$, there exists a conformal factor $\Omega^{2}$ such that $\tilde{g}_{a b}:=\Omega^{2} g_{a b}$ has $X^{a}$ as a KV [11]. By theorem 1, it then follows that a flat, deformed metric $\eta_{a b}$ exists,

$$
\eta_{a b}=\tilde{a} \tilde{g}_{a b}+b H_{a b}
$$

for which $X^{a}$ is a KV, defining next $a:=\Omega^{2} \tilde{a}$ and taking into account the above expression for $\eta_{a b}$ as well as the relation between the metrics $g$ and $\tilde{g}$, it readily follows that $X^{a}$ is a KV of the flat metric

$$
\eta_{a b}=a g_{a b}+b H_{a b}
$$

\section{Examples}

Next we present some physically significant examples. We have chosen families of wellcharacterized spacetimes and then selected, amongst all spacetimes in the family, one well known and physically relevant particular solution. For the sake of convenience, instead of the deformation law (28) in theorem 1, we shall rather use the equivalent formula (7) with the hyperbolic projector $S_{a b}$.

\subsection{Class A1 warped spacetimes}

For these spacetimes, coordinates $x^{a}=u, x^{k}$ with $k=1,2,3$ exist such that the metric takes the following form (see [12] for definitions and further details),

$$
\mathrm{d} s^{2}=\epsilon \mathrm{d} u^{2}+f^{2}(u) h_{i j}\left(x^{k}\right) \mathrm{d} x^{i} \mathrm{~d} x^{j}, \quad \epsilon= \pm 1,
$$

where $f$ is some function of $u$. For $\epsilon=+1, u$ is a spacelike coordinate (class A1 spacelike warped), whereas for $\epsilon=-1, u$ is time (class A1 timelike warped). In what follows, we shall consider only the latter case and put $u:=t$, thus, we shall take the line element to be

$$
\mathrm{d} s^{2}=-\mathrm{d} t^{2}+f^{2}(t) h_{i j}\left(x^{k}\right) \mathrm{d} x^{i} \mathrm{~d} x^{j}, \quad i, j, k=1, \ldots, 3 .
$$

Writing now

$$
\mathrm{d} s^{2}=\tilde{f}^{2}(\tau) \mathrm{d} \tilde{s}^{2}, \quad \text { with } \quad \mathrm{d} \tau=\frac{\mathrm{d} t}{f(t)}, \quad \tilde{f}(\tau)=f(t(\tau))
$$

we get, in an obvious notation,

$$
\mathrm{d} \tilde{s}^{2}=-\mathrm{d} \tau^{2}+p_{i j}\left(x^{k}\right) \mathrm{d} x^{i} \mathrm{~d} x^{j}, \quad \text { or else } \quad g_{a b}=\tilde{f}^{2}(\tau) \tilde{g}_{a b} .
$$


Now, $\partial_{\tau}$ is a KV of $\tilde{g}_{a b}$ and a CKV of the original metric $g_{a b}$; further, it is orthogonally transitive. Hence, $p_{i j}\left(x^{k}\right)$ is a Riemannian metric on the quotient manifold coordinated by $x^{k}, k=1,2,3$.

Making use of the equivalent to the flat deformation theorem in three dimensions for a Riemannian metric (see [13]), we can see that a scalar function $a\left(x^{k}\right)$ and a covariant vector field $\mu_{i}\left(x^{k}\right)$ exist such that they fulfil a previously chosen arbitrary relation, say $\Psi(a,\|\mu\|)=0$, where $\|\mu\|^{2}=p^{i j} \mu_{i} \mu_{j}$, with $p^{i j} p_{j k}=\delta_{k}^{i}$, and the metric

$$
\hat{\eta}_{i j}=a p_{i j}+\mu_{i} \mu_{j}
$$

is flat. Presently, we choose

$$
\Psi(a,\|\mu\|)=\|\mu\|^{2}+a-1=0,
$$

and it then follows that the four-dimensional semi-Riemannian metric

$$
\eta:=-\mathrm{d} \tau \otimes \mathrm{d} \tau+\hat{\eta}_{i j}\left(x^{k}\right) \mathrm{d} x^{i} \otimes \mathrm{d} x^{j}
$$

is also flat and admits the $\mathrm{KV} \partial_{\tau}$.

Using now (75) we have that

$$
\eta:=-\mathrm{d} \tau \otimes \mathrm{d} \tau+a p_{i j} \mathrm{~d} x^{i} \otimes \mathrm{d} x^{j}+\mu_{i} \mathrm{~d} x^{i} \otimes \mu_{j} \mathrm{~d} x^{j},
$$

or else, using the coordinates $x^{a}=x^{1}, x^{2}, x^{3}, x^{4}=\tau$, setting $\mu_{4}=0$ and making use of (73), it turns out that we can write

$$
\eta_{a b}=a \tilde{g}_{a b}-(1-a) \delta_{a}^{4} \delta_{b}^{4}+\mu_{a} \mu_{b}=a \tilde{g}_{a b}+(1-a) S_{a b},
$$

where

$$
S_{a b}:=-\delta_{a}^{4} \delta_{b}^{4}+\hat{\mu}_{a} \hat{\mu}_{b}, \quad \hat{\mu}_{a}:=\frac{1}{\|\mu\|} \mu_{a}
$$

is a two-dimensional hyperbolic projector (recall that we chose $\|\mu\|^{2}=1-a$ ), and thus (76) corresponds the sought for form (7).

\subsection{Spacetimes with additional symmetries}

In some cases with additional symmetries it is possible to derive an explicit expression for $\mu_{i}$; this giving for granted that the deformed metric $\eta_{a b}$, the factors $a$ and $b$, and the hyperbolic projector $S_{a b}$ will share the same additional symmetries. (Note that this is only a conjecture that goes beyond what has been proved so far, although theorem 1 supports its plausibility.)

As an example, take a static spherically symmetric metric

$$
g=-f^{2}(r) \mathrm{d} t \otimes \mathrm{d} t+p^{2}(r) \mathrm{d} r \otimes \mathrm{d} r+r^{2}\left(\mathrm{~d} \theta \otimes \mathrm{d} \theta+\sin ^{2} \theta \mathrm{d} \phi \otimes \mathrm{d} \phi\right) .
$$

which, besides the three KV implementing the spherical symmetry, admits one fourth $\mathrm{KV}$, namely $\partial_{t}$. The quotient space $\mathcal{S}$ can be given the structure of a manifold as discussed previously. Consider next the metric $h$ on $\mathcal{S}$,

$h=g+f^{2}(r) \mathrm{d} t \otimes \mathrm{d} t=p^{2}(r) \mathrm{d} r \otimes \mathrm{d} r+r^{2}\left(\mathrm{~d} \theta \otimes \mathrm{d} \theta+\sin ^{2} \theta \mathrm{d} \phi \otimes \mathrm{d} \phi\right)$.

By the theorem in [13] regarding three-dimensional metrics, a scalar $a$ and a covariant vector $\mu_{i}$ exist, which fulfil an arbitrary, previously chosen constraint, that we shall take $\Psi(a,\|\mu\|):=\|\mu\|^{2}-f^{-2}(r)+a=0$, and are such that the deformed three-dimensional Riemannian metric

$$
\hat{\eta}=a h+\mu \otimes \mu
$$

is flat. Let us next make a guess at $a$ and $\mu$ and take $a=a(r)$ and $\mu=\mu(r) \mathrm{d} r$, we shall have:

$$
\|\mu\|^{2}=h^{i j} \mu_{i} \mu_{j}=p^{-2}(r) \mu^{2}(r),
$$


hence

$$
\hat{\eta}=\left(a+\|\mu\|^{2}\right) p^{2}(r) \mathrm{d} r \otimes \mathrm{d} r+a r^{2}\left(\mathrm{~d} \theta \otimes \mathrm{d} \theta+\sin ^{2} \theta \mathrm{d} \phi \otimes \mathrm{d} \phi\right) .
$$

The spacetime metric $\eta:=-\mathrm{d} t \otimes \mathrm{d} t+\hat{\eta}$ is also flat, i.e.,

$\eta=-\mathrm{d} t \otimes \mathrm{d} t+a h+\mu \otimes \mu=a\left(g+f^{2}(r) \mathrm{d} t \otimes \mathrm{d} t\right)+\mu \otimes \mu-\mathrm{d} t \otimes \mathrm{d} t$,

which is already in the desired form (7) with $b S:=\mu \otimes \mu-\left(f^{-2}(r)-a\right) f^{2}(r) \mathrm{d} t \otimes \mathrm{d} t$.

In order to ensure that $S$ is a hyperbolic projector as required, we need $\|\mu\|^{2}=f^{-2}(r)-a$ which is fulfilled thanks to the chosen arbitrary constraint $\Psi(a,\|\mu\|)=0$.

Substituting the above back into (80) we get that

$$
\hat{\eta}=f^{-2}(r) p^{2}(r) \mathrm{d} r \otimes \mathrm{d} r+a r^{2}\left(\mathrm{~d} \theta \otimes \mathrm{d} \theta+\sin ^{2} \theta \mathrm{d} \phi \otimes \mathrm{d} \phi\right)
$$

must be flat, and this determines $a$ up to a constant. Note that a line element of the form

$$
\mathrm{d} \sigma^{2}=F^{2}(r) \mathrm{d} r^{2}+Y^{2}(r) \mathrm{d} \Omega^{2}
$$

is flat iff

$$
\frac{\mathrm{d} Y(r)}{\mathrm{d} r}= \pm F(r),
$$

hence, choosing the plus sign for convenience and since $Y^{2}=a r^{2}$ and $F(r)=p(r) / f(r)$, we finally get

$\sqrt{a}=\frac{1}{r}\left(\int^{r} \frac{p\left(r^{\prime}\right)}{f\left(r^{\prime}\right)} \mathrm{d} r^{\prime}+K\right), \quad K=$ constant. $\quad$ and $\quad \mu=p(r) \sqrt{f^{-2}(r)-a}$.

Two interesting particular cases are the following:

Friedmann-Robertson-Walker spacetimes. These are particular instances of the ones just discussed, namely: class A1 timelike warped. As is well known, the metric may be written as

$\mathrm{d} s^{2}=-\mathrm{d} t^{2}+\frac{R^{2}(t)}{1+\frac{k}{4} r^{2}}\left(\mathrm{~d} r^{2}+r^{2} \mathrm{~d} \Omega^{2}\right), \quad \mathrm{d} \Omega^{2}=\mathrm{d} \theta^{2}+\sin ^{2} \theta \mathrm{d} \phi^{2}$.

Proceeding as in the general case in section 6.1, we can write $\mathrm{d} s^{2}=R^{2}(t) \mathrm{d} \tilde{s}^{2}$, where

$\mathrm{d} \tilde{s}^{2}:=-\mathrm{d} \tau^{2}+\left(1+\frac{k}{4} r^{2}\right)^{-1}\left(\mathrm{~d} r^{2}+r^{2} \mathrm{~d} \Omega^{2}\right) \quad$ and $\quad \mathrm{d} \tau:=\frac{\mathrm{d} t}{R(t)}$,

with $\partial_{\tau}$ being a $\mathrm{KV}$ of the metric $\tilde{g}$ (of line element $\mathrm{d} \tilde{s}^{2}$ ) and a $\mathrm{CKV}$ of $g$ (line element $\mathrm{d} s^{2}$ ).

The metric $\tilde{g}$ is a particular case of (77) with

$$
f(r):=1 \quad \text { and } \quad p(r):=\left(1+\frac{k}{4} r^{2}\right)^{-1 / 2}
$$

which substituted into (83) yield

$$
\mu=\frac{1-a}{\sqrt{1+k r^{2} / 4}}
$$

and

$$
\sqrt{a}=\frac{1}{r}\left(K+\int^{r} \mathrm{~d} r^{\prime}\left[1+\frac{k}{4} r^{\prime 2}\right]^{-1 / 2}\right) .
$$


Schwarzschild solution. Consider next the well-known Schwarzschild solution written in the form

$g=-\left(1-\frac{r_{s}}{r}\right) \mathrm{d} t \otimes \mathrm{d} t+\left(1-\frac{r_{s}}{r}\right)^{-1} \mathrm{~d} r \otimes \mathrm{d} r+r^{2}\left(\mathrm{~d} \theta \otimes \mathrm{d} \theta+\sin ^{2} \theta \mathrm{d} \phi \otimes \mathrm{d} \phi\right)$,

which is a particular case of (77) with

$$
f(r):=\sqrt{1-\frac{r_{s}}{r}} \quad \text { and } \quad p(r):=1 / f(r)
$$

which substituted into (83) yield

$$
\mu=\sqrt{\left(1-r_{s} / r\right)-a\left(1-r_{s} / r\right)^{2}}
$$

and

$$
\sqrt{a}=1+\frac{r_{s}}{r}\left[K+\ln \left(\frac{r}{r_{s}}-1\right)\right], \quad K=\text { constant }
$$

\section{Acknowledgments}

The authors are grateful to B Coll, A Molina and JM Pozo for interesting comments and suggestions upon reading a previous version of the manuscript. JC acknowledges financial support from the Spanish Ministerio de Educación through grant no. FPA-2007-60220. Partial financial support from the Govern de les Illes Balears is also acknowledged. JLl acknowledges financial support from Ministerio de Educación through grant no. FIS2007-63034 and from the Generalitat de Catalunya, 2001SGR-00061 (DURSI). Both authors are also grateful to an anonymous referee for his comments and suggestions, which have greatly contributed to making the paper more precise and readable.

\section{Appendix A}

We here prove that the constraints (47) propagate out of $\Sigma$. Assume that $a, b$ and $H_{a b}$ is a solution of the reduced PDS (46) for a set of Cauchy data fulfilling the constraints (47) on the Cauchy surface $\Sigma$. We must prove that these constraints also hold on a neighbourhood of $\Sigma$.

Given $a, b$ and $H_{a b}$, consider the metric $\eta_{a b}=a g_{a b}+b H_{a b}$. Let $\nabla$ and $R_{a b c d}$ respectively denote the Levi-Civita connection and the Riemann tensor for $\eta_{a b}$. By the second Bianchi identity we have that

$$
\sum_{\{c d e\}} \nabla_{e} R_{a b c d}:=\nabla_{e} R_{a b c d}+\nabla_{c} R_{a b d e}+\nabla_{d} R_{a b e c} \equiv 0 .
$$

Including (41), the different projections of this equation are

(a) the projection on $X^{b}$ is

$$
\sum_{\{c d e\}}\left(D_{e} L_{c d a}-\frac{1}{2} f_{e} L_{c d a}+\frac{1}{l} L_{a c} \Theta_{e d}-\frac{1}{2} L_{a b c d} \Theta_{e}{ }^{b}\right) \equiv 0,
$$

(b) the totally transverse projection yields

$$
\sum_{\{c d e\}}\left(D_{e} L_{a b c d}+\frac{1}{l} L_{a b c} \Theta_{e d}+\frac{1}{l} L_{c d[a} \Theta_{e b]}\right) \equiv 0,
$$


(c) and the projection on $X^{e}$ is

$$
\nabla_{X} R_{a b c d}+2 \nabla_{[c} R_{a b d] X}-2 R_{a b[d e} \nabla_{c]} X^{e}=0,
$$

which is transverse to $X$ for the indices $c$ and $d$.

As $X^{a}$ is a Killing vector, $\mathcal{L}_{X} R_{a b c d}=0$, and the above equation becomes

$$
\nabla_{[c} R_{a b d] X}-R_{e[b c d} \nabla_{a]} X^{e}=0,
$$

which, projected on $X^{b}$ and including (41), yields

$$
D_{[c} L_{a d]}-\frac{1}{2} L_{a b[d} \Theta_{c]}^{b}-\frac{1}{2} f_{[c} L_{a d]}-\frac{1}{4} L_{c d b} \Theta_{a}{ }^{b}-\frac{l}{4} L_{b a c d} f^{b}=0 .
$$

On its turn, the totally transverse component of (A.5) is

$$
D_{[c} L_{a b d]}-\frac{1}{2} L_{e[b c d} \Theta_{a]}^{e}+\frac{1}{2} L_{c d[b} f_{a]}+\frac{1}{l} \Theta_{[c[b} L_{a] d]}=0 .
$$

In Gaussian normal coordinates equations (A.2), (A.3), (A.6) and (A.7) respectively read

$$
\left.\begin{array}{ll}
\partial_{1} L_{j k a}+2 \partial_{[j} L_{k] 1 a}=\operatorname{lin}, & \partial_{1} L_{a b j k}+2 \partial_{[j} L_{a b k] 1}=\operatorname{lin} \\
\partial_{1} L_{a j}-\partial_{j} L_{a 1}=\operatorname{lin}, & \partial_{1} \partial_{1} L_{a b j}-\partial_{j} L_{a b 1}=\operatorname{lin}
\end{array}\right\}
$$

where $j=2,3$ and $a, b, \ldots=1,2,3$, and 'lin' denotes 'linear terms not containing partial derivatives'. (We have only kept those equations governing the propagation outwards of $\Sigma$, i. e. those containing partial derivatives with respect to $x^{1}$.)

As the metric $\eta_{a b}$ is a solution of the reduced PDS (46), we have that $L_{11}=0, L_{1 j 1}=0$ and $L_{1 i 1 j}=0$. Equations (A.8) thus yield the following linear partial differential system to be fulfilled by the constraints (47):

$$
\begin{array}{ll}
\partial_{1} L_{j k l}=\operatorname{lin}+2 \partial_{[j} L_{k] l 1}, & \partial_{1} L_{j k 1}=\text { lin } \\
\partial_{1} L_{l i j k}=\operatorname{lin}+2 \partial_{[j} L_{l i k] 1}, & \partial_{1} L_{1 i j k}=\text { lin } \\
\partial_{1} L_{1 j}=\operatorname{lin}, & \partial_{1} L_{i j}=\operatorname{lin}+\partial_{j} L_{i 1}
\end{array} \text { and }
$$

which is already in the normal form for the Cauchy-Kowalevski theorem [8]. As the chosen solution $a, b$ and $H_{a b}$ of (46) is assumed to be analytic, the coefficients are analytic. Then, for the Cauchy data $L_{a j}=0, L_{b i j k}=0$ and $L_{j c d}=0$ on $\Sigma$, the solution is unique in the analytic category and, by linearity, $L_{a j}=0, L_{b i j k}=0$ and $L_{j c d}=0$ on an open neighbourhood of $\Sigma$.

\section{Appendix B. The characteristic determinant}

The reduced PDS is constituted by six equations (48), (49) and (51):

$\ddot{l} \cong 0$

$\ddot{m} v_{j}+m \ddot{v}_{j} \cong 0, \quad j=2,3$

$\ddot{a}\left(p_{i j}+[\bar{l}(b+a)-l] v_{i} v_{j}\right)+\ddot{b} \omega_{i} \omega_{j}+b\left[\ddot{\omega}_{i} \omega_{j}+\omega_{i} \ddot{\omega}_{j}\right] \cong 0, \quad i, j=2,3$

where

$$
\ddot{m}=\frac{m}{2}\left(\frac{\ddot{b}+\ddot{a}}{b+a-l / \bar{l}}+\frac{\ddot{a}}{a-l / \bar{l}}\right)
$$

as it easily follows from (38) and (B.1).

The surface $\Sigma$ is non-characteristic if the PDS can be solved for the second partial derivatives of the unknowns, namely $\ddot{a}, \ddot{b}, \ddot{l}, \ddot{v}_{a}$ and $\ddot{\omega}_{b}$ on $\Sigma$, where a 'double dot' stands for 
$\partial_{1}^{2}$. Note that due to the constraints of $p$-unitarity and $p$-orthogonality, in $\ddot{v}_{a}$ and $\ddot{\omega}_{b}$ there are only three independent unknowns. In order to handle them more appropriately we shall consider the $p$-orthonormal triad of spatial covectors

$$
\omega_{a}, v_{a}, \tau_{a} \quad \text { where } \tau_{a}:=\bar{\epsilon}_{a b c} \omega^{b} v^{c},
$$

where $\bar{\epsilon}_{a b c}:=\bar{\epsilon}_{a b c d} X^{d} / \bar{l}$ is the $p$-volume tensor on $\mathcal{S}$.

We then have that

$$
\dot{\omega}_{a}=\Omega_{3} v_{a}-\Omega_{2} \tau_{a}, \quad \dot{v}_{a}=-\Omega_{3} \omega_{a}+\Omega_{1} \tau_{a}, \quad \dot{\tau}_{a}=\Omega_{2} \omega_{a}-\Omega_{1} v_{a}
$$

and, deriving again and keeping only principal terms:

$\ddot{\omega}_{a}=\dot{\Omega}_{3} v_{a}-\dot{\Omega}_{2} \tau_{a}, \quad \ddot{v}_{a}=-\dot{\Omega}_{3} \omega_{a}+\dot{\Omega}_{1} \tau_{a}, \quad \ddot{\tau}_{a}=\dot{\Omega}_{2} \omega_{a}-\dot{\Omega}_{1} v_{a}$

which introduced in (B.2) and (B.3) yields

$\ddot{m} v_{j}-m \omega_{j} \dot{\Omega}_{3}+m \tau_{j} \dot{\Omega}_{1} \cong 0, \quad j=2,3$

$\ddot{a}\left(p_{i j}+[\bar{l}(b+a)-l] v_{i} v_{j}\right)+\ddot{b} \omega_{i} \omega_{j}+2 b v_{(i} \omega_{j)} \dot{\Omega}_{3}-2 b \tau_{(i} \omega_{j} \dot{\Omega}_{2} \cong 0, \quad i, j=2,3$

This last expression (B.6) contains three independent equations, which amount to the contractions with $p^{i j}, \omega^{i} \omega^{j}-p^{i j} \omega^{l} \omega^{l}$ and $v^{i} v^{j}-p^{i j} v^{l} v^{l}$. They read, respectively:

$$
\left.\begin{array}{l}
\left(2+[\bar{l}(b+a)-l] v_{l} v^{l}\right) \ddot{a}+\omega^{l} \omega_{l} \ddot{b}+2 b v^{j} \omega_{j} \dot{\Omega}_{3}-2 b \tau^{j} \omega_{j} \dot{\Omega}_{2} \cong 0 \\
{\left[-\omega^{l} \omega_{l}+[\bar{l}(b+a)-l]\left(\left(v_{l} \omega^{l}\right)^{2}-v_{l} v^{l} \omega^{j} \omega_{j}\right)\right] \ddot{a} \cong 0} \\
-v_{l} \nu^{l} \ddot{a}+\left(\left(v_{l} \omega^{l}\right)^{2}-v_{l} v^{l} \omega^{j} \omega_{j}\right) \ddot{b}-2 b\left(v_{l} \omega^{l} v_{j} \tau^{j}-v_{l} v^{l} \tau^{j} \omega_{j}\right) \dot{\Omega}_{2} \cong 0
\end{array}\right\} .
$$

On its turn, the expression (B.5) consists of two independent equations. They are equivalent to the wedge products with $\tau_{i}$ and $v_{i}$, namely

$$
\left.\begin{array}{l}
-m(v \wedge \omega) \dot{\Omega}_{3}+m(v \wedge \tau) \dot{\Omega}_{1} \cong 0 \\
(\tau \wedge v) \frac{m}{2}\left(\frac{\ddot{b}+\ddot{a}}{b+a-l / \bar{l}}+\frac{\ddot{a}}{a-l / \bar{l}}\right)-m(\tau \wedge \omega) \dot{\Omega}_{3} \cong 0
\end{array}\right\}
$$

where (38) has been used and $(\nu \wedge \omega):=v_{2} \omega_{3}-v_{3} \omega_{2}$ and so on.

Some simplification is gained taking into account that $\left\{\omega_{a}, v_{b}, \tau_{c}\right\}$ is a $p$-orthonormal triad and, in the Gaussian $p$-normal coordinates of section 4.2 , we have that

$$
\omega \wedge v=s \tau_{1} \sqrt{p}, \quad v \wedge \tau=s \omega_{1} \sqrt{p}, \quad \tau \wedge \omega=s v_{1} \sqrt{p}
$$

where $p:=\operatorname{det}\left(p_{i j}\right)$, and

$$
\left(\omega^{l} v_{l}\right)^{2}-\omega^{l} \omega_{l} v^{j} v_{j}=-\frac{1}{p}(v \wedge \omega)^{2}=-v_{1}^{2}, \quad \omega^{l} v_{l} v^{j} \tau_{j}-\omega^{l} \tau_{l} v^{j} v_{j}=\omega_{1} \tau_{1} .
$$

Furthermore,

$$
\omega^{l} \omega_{l}=1-s \omega_{1}^{2}, \quad v^{j} \tau_{j}=-s v_{1} \tau_{1}, \quad \omega^{j} \tau_{j}=-s \omega_{1} \tau_{1} .
$$

Substituting this into (B.1), (B.7) and (B.8), we obtain

$$
\left.\begin{array}{l}
\left(2+[\bar{l}(b+a)-l]\left(1-s v_{1}^{2}\right)\right) \ddot{a}+\left(1-s \omega_{1}^{2}\right) \ddot{b}-2 s b v_{1} \omega_{1} \dot{\Omega}_{3}+2 s b \tau_{1} \omega_{1} \dot{\Omega}_{2} \cong 0 \\
{\left[-1+s \omega_{1}^{2}-[\bar{l}(b+a)-l] v_{1}^{2}\right] \ddot{a} \cong 0} \\
-\left(1-s v_{1}^{2}\right) \ddot{a}-v_{1}^{2} \ddot{b}-2 b \omega_{1} \tau_{1} \dot{\Omega}_{2} \cong 0 \\
m s \tau_{1} \sqrt{p} \dot{\Omega}_{3}+m s \omega_{1} \sqrt{p} \dot{\Omega}_{1} \cong 0 \\
-\frac{m}{2} s \omega_{1} \sqrt{p}\left(\frac{\ddot{b}+\ddot{a}}{b+a-l / \bar{l}}+\frac{\ddot{a}}{a-l / \bar{l}}\right)-m s v_{1} \sqrt{p} \dot{\Omega}_{3} \cong 0
\end{array}\right\}
$$


The reduced PDS (B.1)-(B.3) can be solved for all the second partial derivatives of the unknowns, namely $\ddot{a}, \ddot{b}, \ddot{l}, \ddot{v}_{a}$ and $\ddot{\omega}_{b}$, if, and only if, the system (B.9) can be solved for the six unknowns $\ddot{a}, \ddot{b}, \ddot{l}, \dot{\Omega}_{1}, \dot{\Omega}_{2}$ and $\dot{\Omega}_{3}$; that is if, and only if, it has a non-null determinant, $\Delta \neq 0$, where

$$
\begin{aligned}
\Delta:=2 b \omega_{1}^{2} v_{1} \tau_{1} p & {\left[1-s \omega_{1}^{2}+[\bar{l}(b+a)-l] v_{1}^{2}\right] \frac{l-a \bar{l}}{l^{2}} } \\
\times & {\left[\left(b+a-\frac{l}{\bar{l}}\right)\left(1-s v_{1}^{2}\right)-s \omega_{1}^{2}\left(a-\frac{l}{\bar{l}}\right)\right], }
\end{aligned}
$$

which stands for the characteristic determinant of the partial differential system (B.1)-(B.3).

\section{References}

[1] Llosa J and Soler D 2005 Class. Quantum Grav. 22 893-908

[2] Coll B, Hildebrand S R and Senovilla J M M 2001 Gen. Rel. Grav. 33649

[3] Kobayashi S and Nomizu K 1969 Foundations of Differential Geometry (New York: Interscience)

[4] Geroch R 1971 J. Math. Phys. 12 918-24

[5] Beig R and Schmidt B G 2000 Time independent gravitational fields Einstein's Field Equations and Their Physical Implications (Lecture Notes in Physics vol 540) ed B G Schmidt (Berlin: Springer) pp 325-72

[6] Stefani H, Kramer D, MacCallum M, Hoenselaers C and Herlt E 2003 Exact Solutions of Einstein's Field Equations (Cambridge: Cambridge University Press)

[7] Friedrich H and Rendall A 2000 The Cauchy problem for the Einstein equations Einstein's Field Equations and Their Physical Implications (Lecture Notes in Physics vol 540) ed B G Schmidt (Berlin: Springer) pp 127-224

[8] John F 1982 Partial Differential Equations (Berlin: Springer)

[9] Hicks N J 1965 Notes on Differential Geometry (Princeton, NJ: Van Nostrand-Reinhold)

[10] Choquet-Bruhat Y, DeWitt-Morette C and Dillard-Bleick M 1987 Analysis, Manifolds and Physics (Amsterdam: North-Holland)

[11] Hall G S 2004 Symmetries and Curvature Structure in General Relativity (Lecture Notes in Physics vol 46) (Singapore: World Scientific)

[12] Carot J and da Costa J 1993 Class. Quantum Grav. 10 461-82

[13] Coll B, Llosa J and Soler D 2002 Gen. Rel. Grav. 34 269-82 